\title{
Hypertension and meditation: can meditation be useful in preventing hypertension?
}

\author{
Yadneshwar Khobragade*, Sujata Khobragade, Adinegara bin Lutfi Abbas
}

\begin{abstract}
Department of Community Medicine, Melaka-Manipal Medical College (MMMC), Jalan Batu Hampar, Bukit Baru, Melaka-75150, Malaysia
\end{abstract}

Received: 03 May 2016

Accepted: 04 June 2016

\section{*Correspondence:}

Dr. Yadneshwar Khobragade,

E-mail: drkhobragade@yahoo.com

Copyright: ( $)$ the author(s), publisher and licensee Medip Academy. This is an open-access article distributed under the terms of the Creative Commons Attribution Non-Commercial License, which permits unrestricted non-commercial use, distribution, and reproduction in any medium, provided the original work is properly cited.

\begin{abstract}
Hypertension is elevation of systolic blood pressure equal to or greater than $140 \mathrm{~mm}$ of $\mathrm{Hg}$ and or diastolic blood pressure of (fifth Korotkoff's Sound) equal to or greater than $90 \mathrm{~mm}$ of $\mathrm{Hg}$. Globally prevalence of hypertension is $26 \%$ to $30 \%$. Essential hypertension constitutes more than 95 percent of all hypertensive cases and is influenced by unhealthy diet, obesity, sedentary life style, emotions and persistent exposure to stress. Hypertension is major cause of cardiovascular mortality and morbidity. It is also said to be the "Silent Killer" because of its sudden appearance in the form of cardiovascular complications such as myocardial infarction or stroke. Meditation is a set of techniques that are intended to encourage a heightened state of awareness and focussed attention. Practitioner of meditation brings about desirable changes in their thoughts, perceptions, behaviour and lifestyle. Increased stress and hostility has been associated with increased release of catecholamine and corticoids causing hypertension and its complications. Meditation helps in changing attitude and approach towards life. It inculcates right thoughts and behaviour. Physiologically meditation causes parasympathetic dominance over sympathetic; maintain pulse rate and blood pressure, reduces vascular spasm and prevents complications associated with hypertension. After going through abstracts of 55 studies, we reviewed 10 randomized controlled trials which were compared by combining meditation with meditation and medication or meditation alone in patients above the age of 18 years, both men and women having blood pressure greater than 140/90mm of $\mathrm{Hg}$ doing meditation under supervision of meditation teacher or health care worker having trained in meditation. Meditation studies in any setting were considered. Control group included either people on medication or without medication or not doing meditation. Clustered and crossover studies and controlled after and before trials were excluded. Trials were considered where follow up period was eight weeks or more. Studies show that meditation improves exercise tolerance of stress test, reduces pulse rate, heart rate, stroke volume and blood pressure. Regular practice of meditation keeps the blood pressure under control hence reduction in all-cause mortality, non-fatal Myocardial Infarction and stroke. In view of the beneficial effects of meditation, it may be useful as primary intervention strategy in prevention of hypertension.
\end{abstract}

Keywords: Meditation, Healthy lifestyle, Hypertension, Equanimeous, Non-judgemental, Mindfulness, Hostility, Stress, Anxiety

\section{INTRODUCTION}

Hypertension means systolic blood pressure greater than $140 \mathrm{~mm}$ of $\mathrm{Hg}$ and or diastolic blood pressure greater than $90 \mathrm{~mm}$ of $\mathrm{Hg}$. If blood pressure is uncontrolled, it can lead myocardial infarction, stroke, visual loss or chronic kidney disease. More than 95 percent of hypertensives have essential hypertension and contributory factors for this are family history, ageing, obesity, smoking, unhealthy diet, emotional and chronic psychosocial stress. The global prevalence of hypertension ranges from 22 to $33 \%$. 
In Malaysia, India and USA prevalence of hypertension is $12-26 \%, 26.2 \%$ and $28-30 \%$ respectively. ${ }^{1-3}$ Because hypertension causes extensive cardiovascular morbidity and mortality and increased cost of health care; various modalities of preventive measures are being promoted. Studies on Meditation have shown that it helps in changing lifestyle, behavior and outlook towards life and may be effective in management of hypertension as a primary preventive strategy.

Meditation is a means of transforming the human mind. Meditation practices are techniques that encourage and develop concentration, clarity, emotional positivity, and serenity. Person is able to observe true nature of physical structure of body and mind. By engaging with a particular meditation practice person learns the patterns and habits of his/her mind, and the practice offers a means to change the behavior and cultivate new and more positive ways of life.

With regular meditative practice and patience these nourishing, focused states of mind can deepen into profoundly peaceful and energized states of mind. Such experiences can have a transformative effect and can lead to a new understanding of life. ${ }^{4}$ Regular practice of meditation helps meditator to develop self-control, right attitude and behaviour, and ability to cope up with situation. $^{1,5}$

Our thoughts and behaviour influence our physical activities, emotions and interaction with others. Negative emotions such as anger, hatred, ill will, animosity may accelerate blood pressure and person may develop ischaemic heart disease, myocardial infarction and stroke. $^{2-9}$ At hormonal level mental stress increases release of catecholamine, corticoids and thyroxine which are strongly associated with rise in blood pressure both systolic and diastolic. ${ }^{6,7,10}$

Cardiovascular diseases carry highest mortality among all cause deaths $(23 \%)$. Worldwide $30 \%$ of all the deaths (2001) have been due to CVS, of them $50 \%$ are in developed and $28 \%$ in developing and underdeveloped countries (Mathers and others 2001; WHO 2002a). More than 9.4 million (12.8\% of all deaths) people die due to hypertension and 57 million people suffer from disability adjusted life years. $^{11,12}$

Regular practise of meditation reduces catecholamine, adrenal-cortical hormones and may help reducing blood pressure. ${ }^{13,14}$ Thus meditation can be useful as primary intervention strategy to prevent development of hypertension and its complications.

In order to find out effectiveness of meditation in hypertension; we conducted this systematic review with following objectives to determine the effectiveness of meditation in patients with hypertension, to determine the effectiveness of meditation in reducing morbidity and mortality due hypertension and its complications and to study the effect of meditation on general well-being and lifestyle modifications.

\section{Types of studies taken for this review}

All Randomised Controlled Trials were compared by combination of meditation and meditation with medication or meditation alone. Cluster-randomised trials, Controlled before and after trials and cross over trials were excluded.

\section{Types of participants}

People with hypertension of ages 18 years and above, both sexes, in any setting were included.

\section{Type of interventions}

Meditation Therapy designed to reduce hypertension in participants with hypertension were considered. All nonpharmacological Meditation Therapy interventions delivered by health care workers with specific training in these techniques were considered. Comparison group were either people without medication or no meditation or medication alone. Trials were only considered where the follow up was 8 weeks or more following the start of the intervention.

\section{Types of outcome measures}

While the interventions is a mind control technique causing reduced sympathetic tone and increased parasympathetic dominance leading to relaxation of smooth muscle fibres of cardiovascular system thereby preventing hypertension, and death.

\section{Primary outcomes}

Measuring reduction in blood pressure and mortality due to cardiovascular disease specially Hypertension, Time for follow up period of primary outcome measures was 2 months or above.

\section{Secondary outcomes}

- To see if any adverse effect occurs due to meditation.

- To see if it helps in preventing prolonged stay in hospital or reduction in intake of medicine or reduction in cost of health care.

\section{Search methods for identification of studies}

\section{Electronic searches}

We searched Medline, EMBASE, Central on the Cochrane Library, LILACS (Virtual health library of Latin American countries). There was no language barrier for searching articles. Well debated key terms were 
included in the search strategy. Other search engines that were utilized for extraction of relevant electronic data from the internet include: PUBMED, GOOGLE SCHOLAR, EMBASE, Clinicaltrials.gov, Meta Register of Controlled Trials on http://www.controlledtrials.com/mrct/ and WHO ICTRP Search Portal on http://apps.who.int/trialsearch/

Other websites that were used - www.vri.dhamma.org; http://www.rccm.org.uk; http://nccam.nih.gov

\section{Techniques of meditation}

- In mindfulness Meditation subjects are asked to sit comfortably in an environment of reduced illumination with closed eyes and instructed to focus their mind on incoming and outgoing breath in unattached and unbiased manner. With intense concentration meditator is able to control his excessive distracting thoughts. For beneficial effects meditators are advised to do meditation for 1 hour daily in morning and evening.

- In Vipassana meditation subjects learn the technique under the guidance of a vipassana teacher. It is a residential course of 10 days in which meditators are asked to observe moral precepts and keep complete silence for the entire duration of course. It runs from morning 4.30 to evening 9.30 with half an hour break each for breakfast, lunch and evening tea. Meditation is done in poorly illuminated room in comfortable sitting position with spine straight and eyes closed. It is taught in 3 steps. First Meditators learn observation of breath (ana-panna) meditation for three and half days. Second step is observation of bodily sensation systematically in unbiased, unattached and non-judgemental manner for another six and half day. Throughout the process of meditation, an individual remains awake and aware of sensations occurring in body at that particular moment. Third step is meditation of loving kindness or sharing of merits and extending good wishes to others. The ultimate aim of meditation is to develop calm, quite, stable and Equanimeous or balanced mind, remove negativities like anger, hatred, ill will, animosity and fill it with positive thoughts like love, compassion and goodwill for self and others. ${ }^{15}$ The teachers in vipassana meditation advocate practice of this technique for one hour in morning and one hour in evening on regular basis for beneficial effects. For observation of changes in mind, body and behaviour minimum one year of regular practice is desirable.

- In Transcendental meditation (TM), subjects are asked to chant mantra in calm and quiet place with closed eyes for 30 minutes each in the morning and evening. It causes relaxation of mind and body. For better results meditators are advised not to disclose their mantra to others.
- Islamic meditation: reference has been made about meditative practices in Islam for spiritual growth of an individual. It is stated by Prophet Muhammad that "One hour of meditation is more valuable than seventy years of obligatory worship." Zikr or Islamic meditation advocates meditation in sitting position, comfortably in calm and serene place. Stages include (a) Tasbih (chanting or recitation of Qur'an), (b) Muraqaba (observation and surrender to Allah), (c) Taffakur (think and contemplate), (d) Murabita (connection with Allah). This helps relaxing mind and body. In order to have better understanding and spiritual development, it is advised to do meditation for minimum 30 minutes morning and evening atleast 5 days a week. Similarly fasting and asking for forgiveness during the month of Ramadan and sharing one's merit and wealth (Zalat) dissolves ego and have been found to have positive impact on health. ${ }^{16}$

- Zen or Zazen Meditation: Here subjects are taught to take full lotus or semi lotus position. Spine is kept straight and position has to be comfortable with closed or semi-closed eyes. Initially mind is focused on breath and after a week of practice one is asked to quieten the mind. However when distracting thought appear in mind the meditators are asked to observe without reacting or getting carried away with thoughts. A stage comes when mind becomes calm, quiet and peaceful. One is expected to practise meditation daily for at-least 30 minutes.

- In all the above meditation practices meditators are given discourses which guide a meditator to have right type of diet, right type of thoughts, right type attitude and right type of behaviour which are beneficial to self and others. The mastery of an individual meditation technique depends on its respective philosophical school of thought. The meditation procedure is usually communicated under the supervision of an experienced meditation teacher. Here different phenomenological systems of thought provide elaborate cognitive instructions that lead to the control of specific physiological states. ${ }^{17}$

The main purpose of meditation developed by various religious schools of thought is for the spiritual growth of an individual, which can help in leading a peaceful, happy and contented life.

\section{Analysis}

Meta-analysis was done using Practical Meta-Analysis Effect Size Calculator David B. Wilson, Ph.D., George Mason University.

\section{RESULTS}

We have searched electronic databases and found more than 55 articles, abstracts and clinical trials studies. Most 
of the studies were on Transcendental meditation ${ }^{\mathrm{TM}}$ on cardiovascular diseases. Some studies using complimentary therapies include meditation along with diet modification and lifestyle changes. A prospective randomised study conducted by Jose Antonia Curiat et al, published in J. alt. and compli Med Brazil on 19 patients found significantly reduced production of norepinephrine in meditation group $(677.7 \pm 96.6 \mathrm{pg} / \mathrm{ml})$ compared to control $(387.1 \pm 39.1 \mathrm{pg} / \mathrm{ml})$, reduced VE / $\mathrm{VCO} 2$ slope and positive impact on congestive heart failure. ${ }^{18}$ We did not find any specific study on Islamic meditation in hypertension but reduction in heart rate and blood pressure has been noticed with practice of Salat, an Islamic form of meditation. ${ }^{19}$ Search under www.vri.org and other search engine stated above did not find any study of Vipassana meditation on hypertension but we could find mindfulness based studies which are one of the components of Vipassana Meditation. We could find some relevant and related studies on meditation in hypertension as shown below:

Table 1: Effect of meditation on hypertension.

\begin{tabular}{|c|c|c|c|c|}
\hline Type of meditation & Type of study & $\begin{array}{l}\text { Sample size and BP } \\
\text { cut off } \mathrm{Pt} \text {. }\end{array}$ & Duration of study & Results \\
\hline $\begin{array}{l}\text { Transcendental meditation } \\
\text { hypertension in older Afric } \\
\text { Americans. ( USA). }{ }^{37}\end{array}$ & $\begin{array}{l}\text { A randomised controlled trial TM } \\
\text { compared with lifestyle } \\
\text { modification program }\end{array}$ & $\begin{array}{l}127 \text { men and women } \\
\text { (55-85 years) with } \\
\text { diastolic BP } 90 \text { to } 109 \\
\mathrm{~mm} \mathrm{Hg} \text { and SBP } \\
<189 \mathrm{~mm} \mathrm{Hg} \text { (baseline } \\
\mathrm{BP}=<\text { or }=179 / 104 \mathrm{~mm} \\
\mathrm{Hg}) .\end{array}$ & 4 months & $\begin{array}{l}\text { TM reduced systolic pressure by } \\
10.7 \mathrm{~mm} \mathrm{Hg}(\mathrm{P}<0.0003) \text { and } \\
\text { diastolic pressure by } 6.4 \mathrm{~mm} \mathrm{Hg} \\
(\mathrm{P}<0.00005) 0.0003) \text { compared } \\
\text { to lifestyle modification }\end{array}$ \\
\hline $\begin{array}{l}\text { Stress reduction by TM } \\
\text { in hypertension (USA). }\end{array}$ & $\begin{array}{l}\text { A randomized controlled trial in } \\
\text { African-Americans }\end{array}$ & $\begin{array}{l}\text { men and women }(N= \\
150, \text { mean age } 49 \pm 10 \\
\text { years, mean blood } \\
\text { pressure }(\mathrm{BP})=142 / 95 \\
\mathrm{~mm} \mathrm{Hg}) \text { in urban } \\
\text { community health } \\
\text { centre }\end{array}$ & 1 year & $\begin{array}{l}\mathrm{TM}-\text { Reduction in } \\
\mathrm{SBP} / \mathrm{DBP}=3.11 / 5.7 \mathrm{~mm} \mathrm{Hg} \text { and } \\
\text { reduction in medication }\end{array}$ \\
\hline $\begin{array}{l}\text { Long-term effects of } \\
\text { stress reduction } \\
\text { transcendental } \\
\text { meditation) on mortality } \\
\text { in persons } \geq 55 \text { years of } \\
\text { age with systemic } \\
\text { hypertension (USA). }\end{array}$ & $\begin{array}{l}\text { Randomised control trials } \\
\text { hypertensives above } 55 \text { years }\end{array}$ & $\begin{array}{l}202 \text { subjects, including } \\
77 \text { whites (mean age } \\
81 \text { years) and } 125 \\
\text { African-American } \\
\text { (mean age } 66 \text { years) } \\
\text { men and women. }\end{array}$ & $\begin{array}{l}\text { Follow-up max } 18.8 \\
\text { years (Mean } 7.6+/- \\
3.5 \text { years) for vital } \\
\text { status and cause of } \\
\text { death determined } \\
\text { from the national } \\
\text { death index. }\end{array}$ & $\begin{array}{l}23 \% \text { decrease in all-cause } \\
\text { mortality and } 30 \% \text { in } \\
\text { cardiovascular mortality in TM } \\
\text { group compared to control }\end{array}$ \\
\hline $\begin{array}{l}\text { Transcendental } \\
\text { meditation in } \\
\text { hypertension (USA). }{ }^{40}\end{array}$ & Randomised control trial & $\begin{array}{l}\text { Meditation training } \\
\text { and essential } \\
\text { hypertension, No. of } \\
\text { pt. } 41,23 \mathrm{M}: 18 \mathrm{~F}\end{array}$ & 3 months & Decrease blood pressure \\
\hline $\begin{array}{l}\text { Mindfulness based } \\
\text { meditation in } \\
\text { hypertension (evaluation } \\
\text { of a mindfulness-based } \\
\text { intervention program to } \\
\text { decrease blood pressure } \\
\text { in low-income african- } \\
\text { american older adults). } \\
\text { (USA). }\end{array}$ & Randomised control trial & $\begin{array}{l}20 \text { hypertensive } \\
\text { patients on medication } \\
>64 \text { years } \\
19 \mathrm{~F}, 1 \mathrm{M}\end{array}$ & 2 months & $\begin{array}{l}\text { Reduction in SBP by } \\
21.92 \mathrm{mmHg} \text { and DBP by } \\
16.70 \mathrm{mmHg} \text { compared to control } \\
\text { who were getting social support }\end{array}$ \\
\hline $\begin{array}{l}\text { Contemplative } \\
\text { meditation reduces } \\
\text { ambulatory blood } \\
\text { pressure and stress- } \\
\text { induced hypertension: a } \\
\text { randomized pilot trial } \\
\text { (Germany). }\end{array}$ & Randomised control trial & $\begin{array}{l}52 \text { cases of untreated } \\
\text { hypertension }\end{array}$ & 2 months & $\begin{array}{l}\text { Reduction in heart rate by } 13 \text { and } \\
\text { systolic blood pressure by } \\
15 \mathrm{mmHg} \text { and diastolic BP by } 13 \\
\text { compared to } 3 \text { and } 2 \mathrm{mmHg} \text { in } \\
\text { control respectively. }\end{array}$ \\
\hline $\begin{array}{l}\text { Mindfulness meditation } \\
\text { in hypertension } \\
\text { (Canada) } \\
\text { (analysis of stress } \\
\text { reduction using } \\
\text { mindfulness meditation } \\
\text { and yoga: results from } \\
\text { the harmony randomized } \\
\text { controlled trial). }\end{array}$ & $\begin{array}{l}\text { Harmony randomised controlled } \\
\text { trial in mild hypertension (no } \\
\text { medication) }\end{array}$ & $\begin{array}{l}\text { 101adults participants } \\
(\mathrm{Men}=38 ; \\
\text { Women=63) }\end{array}$ & $\begin{array}{l}2 \text { months practise of } \\
\text { meditation and its } \\
\text { effect on HTN. }\end{array}$ & $\begin{array}{l}\text { Slight reduction in BP in women } \\
\text { practising meditation Compared } \\
\text { to control. Statist no significant } \\
\text { difference between meditators } \\
\text { and control. }\end{array}$ \\
\hline $\begin{array}{l}\text { Mindfulness meditation } \\
\text { and Jacobson's } \\
\text { Progressive muscle } \\
\text { relaxation (PMR), in }\end{array}$ & $\begin{array}{l}\text { Quasi-experimental } \\
\text { cardiac rehabilitation programme } \\
\text { in iran }\end{array}$ & $\begin{array}{l}\text { No. of patients } 45 \\
(\mathrm{M}=18, \mathrm{~F}=27) \text {, with } \\
\text { CVD and depression; } \\
15 \text { Meditation, }\end{array}$ & & $\begin{array}{l}\text { Reduction in SBP, DBP and } \\
\text { heart rate in meditators } \\
\text { compared to relaxation technique }\end{array}$ \\
\hline
\end{tabular}




\begin{tabular}{|c|c|c|c|c|}
\hline $\begin{array}{l}\text { CVD (Comparison of } \\
\text { Cardiac Rehab. } \\
\text { Programs Combined } \\
\text { with Relaxation and } \\
\text { Meditation Techniques). } \\
\text { (Iran). }{ }^{21}\end{array}$ & & $\begin{array}{l}15 \text { relaxation and } 15 \\
\text { control, age } 45-65 \\
\text { years }\end{array}$ & & \\
\hline $\begin{array}{l}\text { Zen meditation and } \\
\text { hypertension } \\
\text { (Decrease in blood } \\
\text { pressure and improved } \\
\text { psychological aspects } \\
\text { through meditation } \\
\text { training in hypertensive } \\
\text { older adults) (Brazil). }{ }^{20}\end{array}$ & Randomised controlled trial & $\begin{array}{l}\text { A total of } 59 \\
\text { volunteers ( } 21 \text { men } \\
\text { and } 38 \text { women), aged } \\
\geq 60 \text { years with SBP } \\
\text { between } 130 \text { and } \\
159 \mathrm{mmHg} \text { and DBP } \\
\text { between } 85 \text { and } \\
99 \mathrm{mmHg} \text {, meditation } \\
\text { group } \mathrm{n}=28 \text { and a } \\
\text { control group } \mathrm{n}=31\end{array}$ & 3 months & $\begin{array}{l}\text { Reduction in SBP, } \\
\text { psychologically better and } \\
\text { improved quality of life. }\end{array}$ \\
\hline $\begin{array}{l}\text { Dhammakaya (samata) ) } \\
\text { meditation (Thailand). }{ }^{24}\end{array}$ & $\begin{array}{l}\text { Selective sample of students 20-25 } \\
\text { years }\end{array}$ & $\begin{array}{l}\text { Expt. Group- } 52 \text { males } \\
\text { stayed as monks in } \\
\text { monastery Control } \\
\text { group } 30 \text { males stayed } \\
\text { at home. }\end{array}$ & 6 Weeks & $\begin{array}{l}\text { Decrease in serum cortisol, syst. } \\
\text { and diast. BP, vital capacity, } \\
\text { tidal vol., reaction time and } \\
\text { increase serum protein }\end{array}$ \\
\hline $\begin{array}{l}\text { Catecholamine levels in } \\
\text { practitioners of the } \\
\text { transcendental } \\
\text { meditation technique } \\
(\text { USA })^{28}\end{array}$ & $\begin{array}{l}\text { Experiment, estimation of } \\
\text { norepinephrine and epinephrine }\end{array}$ & $\begin{array}{l}\text { Healthy subjects, } \\
\text { Study grp practising } \\
\text { TM. }=19 \\
\text { Control }=16\end{array}$ & $\begin{array}{l}2-15 \text { years, average } \\
7 \text { years }\end{array}$ & $\begin{array}{l}\text { Reduction in morning and } \\
\text { evening levels of norepinephrine } \\
\text { and epinephrine in TM } \\
\text { compared to control }\end{array}$ \\
\hline $\begin{array}{l}\text { Meditation reduces } \\
\text { sympathetic activation } \\
\text { and improves the quality } \\
\text { of life in elderly patients } \\
\text { with optimally treated } \\
\text { heart failure: a } \\
\text { prospective randomized } \\
\text { study. (Brazil) }\end{array}$ & $\begin{array}{l}\text { Estimation of Nor-epinephrine } \\
\text { levels }\end{array}$ & $\begin{array}{l}\text { Optimally treated } \\
\text { patients of } \mathrm{CCF}\end{array}$ & $\begin{array}{l}3 \text { months } \\
\text { mindfulness } \\
\text { meditation }\end{array}$ & $\begin{array}{l}\text { Reduction in level of NE from } \\
677.7+/-96.6 \text { to } 387.1+/-39.1 \\
\text { pg/mL (p }=0.008) \text { in } \mathrm{M} \text { versus } \\
491.4+/-35.9 \text { to } 470.6+/-31.2 \\
(\mathrm{p}=0.34) \text { in } \mathrm{C} \text {; }\end{array}$ \\
\hline $\begin{array}{l}\text { Islamic meditation } \\
(\text { Malaysia) })^{\mathbf{3 8}}\end{array}$ & Selective sampling & $\begin{array}{l}30 \text { male adult } \\
\text { participants ( } 20-30 \\
\text { years) }\end{array}$ & & $\begin{array}{l}\text { Reduction in systoilic blood } \\
\text { pressure of } 3 \mathrm{mmHg} \text { and } \\
\text { diastolic by } 2 . \mathrm{mmHg} \& \text { heart } \\
\text { rate by } 3 / \mathrm{minute}\end{array}$ \\
\hline
\end{tabular}

Table 2: Systolic and diastolic blood pressure changes after meditation compared to control.

\begin{tabular}{|c|c|c|c|c|c|c|c|c|c|c|c|c|c|}
\hline $\begin{array}{l}\text { Author } \\
\text { Name }\end{array}$ & Title & $\begin{array}{l}\text { Duration } \\
\text { of study }\end{array}$ & $\begin{array}{l}\text { Sampli } \\
\text { size }\end{array}$ & $\begin{array}{l}\text { Study } \\
\text { group/ } \\
\text { control }\end{array}$ & $\begin{array}{l}\text { Male/ } \\
\text { female }\end{array}$ & Age & $\begin{array}{l}\text { Cut off } \\
\text { point (mm Hg) }\end{array}$ & $\begin{array}{l}\text { SBP } \\
\text { (B) }\end{array}$ & $\begin{array}{l}\text { SBP } \\
(\mathbf{A})\end{array}$ & $\begin{array}{l}\text { SBP } \\
(\mathbf{B}-\mathbf{A})\end{array}$ & $\begin{array}{l}\text { DBP } \\
\text { (B) }\end{array}$ & $\mathbf{D B P}(\mathbf{A})$ & $\begin{array}{l}\text { DBP } \\
(\mathbf{B}-\mathbf{A})\end{array}$ \\
\hline $\begin{array}{l}\text { Schneider } \\
\text { RH et al }\end{array}$ & TM in HTN & 4 months & 74 & $\begin{array}{l}36 \\
38\end{array}$ & Both & $\begin{array}{l}55- \\
85\end{array}$ & $\begin{array}{l}>140 / 90 \\
\text { to } 180 / 110\end{array}$ & $\begin{array}{l}179 \\
150 \\
\end{array}$ & $\begin{array}{l}168 \\
148 \\
\end{array}$ & $\begin{array}{l}11 \\
2\end{array}$ & $\begin{array}{l}104 \\
94 \\
\end{array}$ & $\begin{array}{l}94 \\
92\end{array}$ & $\begin{array}{l}10 \\
2\end{array}$ \\
\hline $\begin{array}{l}\text { Schneider } \\
\text { RH et al }\end{array}$ & TM in HTN & 12 months & 106 & $\begin{array}{l}54 \\
52 \\
\end{array}$ & Both & $>50$ & $>140 / 95$ & $\begin{array}{l}142 \\
144.4 \\
\end{array}$ & $\begin{array}{l}139 \\
143.5 \\
\end{array}$ & $\begin{array}{l}3 \\
0.9\end{array}$ & $\begin{array}{l}95 \\
96\end{array}$ & $\begin{array}{l}89 \\
95 \\
\end{array}$ & $\begin{array}{l}6 \\
1 \\
\end{array}$ \\
\hline $\begin{array}{l}\text { Priya } \\
\text { Palta et al }\end{array}$ & $\begin{array}{l}\text { MM in afro- } \\
\text { Am low } \\
\text { income Gp. } \\
\text { Include pt } \\
\text { on medicine }\end{array}$ & 2 months & 20 & $\begin{array}{l}12 \\
8\end{array}$ & $\begin{array}{l}1 \\
19\end{array}$ & $>62$ & & $\begin{array}{l}148 \\
144\end{array}$ & $\begin{array}{l}137 \\
140\end{array}$ & $\begin{array}{l}11 \\
4\end{array}$ & $\begin{array}{l}78 \\
67\end{array}$ & $\begin{array}{l}74 \\
70\end{array}$ & $\begin{array}{l}4 \\
\text { Rise } 3\end{array}$ \\
\hline $\begin{array}{l}\text { JP } \\
\text { Manikon } \\
\text { da et al }\end{array}$ & $\begin{array}{l}\text { Contemplati } \\
\text { ve } \\
\text { meditation } \\
\text { and Breath } \\
\text { Technique } \\
\text { in HTN }\end{array}$ & 2 months & 49 & $\begin{array}{l}26 \\
23\end{array}$ & $\begin{array}{l}23 \\
26\end{array}$ & & $\begin{array}{l}>140 / 85 \text { to } \\
180 / 110\end{array}$ & & & $\begin{array}{l}15 \\
3\end{array}$ & & & 13 \\
\hline $\begin{array}{l}\text { Kimberl } \\
\mathrm{y} \text { et al }\end{array}$ & MM in HTN & 3 months & 101 & $\begin{array}{l}51 \\
50\end{array}$ & $\begin{array}{l}38 \\
63\end{array}$ & $\begin{array}{l}>20- \\
75\end{array}$ & $\begin{array}{l}\text { Amb. BP } \\
135 / 85 \\
24 \mathrm{hrs} \\
>130 / 80\end{array}$ & $\begin{array}{l}140 \\
--\end{array}$ & $\begin{array}{l}139 . \\
6\end{array}$ & $\begin{array}{l}.4 \pm \\
6.7 \\
.4 \pm \\
7.8\end{array}$ & $\begin{array}{l}87 \\
--\end{array}$ & $\begin{array}{l}86.6 \\
--\end{array}$ & $\begin{array}{l}0.0 \pm 4 . \\
9 \\
0.0 \pm \\
4.6\end{array}$ \\
\hline $\begin{array}{l}\text { Marcia } \\
\text { de } \\
\text { Fatima } \\
\text { Rosas } \\
\text { Marchio } \\
\text { ri et al }\end{array}$ & $\begin{array}{l}\text { Zen Meditatior } \\
\text { in HTN. } \\
\text { Patients } \\
\text { stabilized on } \\
\text { drugs included }\end{array}$ & 3 months & 59 & $\begin{array}{l}28 \\
31\end{array}$ & $\begin{array}{l}21 \\
38\end{array}$ & $>60$ & $\begin{array}{l}130 / 85 \text { to } \\
160 / 100\end{array}$ & & & & & & \\
\hline Mahdy & $\mathrm{MM}$ in CVD & 2 months & 45 & MM 15 & 18 & $>45$ & & 134 & 108 & 26 & 72 & 67 & 5 \\
\hline
\end{tabular}




\begin{tabular}{|c|c|c|c|c|c|c|c|c|c|c|c|c|c|}
\hline $\begin{array}{l}\text { Hasanza } \\
\text { dah }\end{array}$ & $\begin{array}{l}\text { and depression } \\
\text { (cardiac } \\
\text { rehabilitation } \\
\text { prog) }\end{array}$ & & & $\begin{array}{l}\text { PMR } 15 \\
\text { CTR } 15\end{array}$ & 27 & 65 & & $\begin{array}{l}126 \\
111\end{array}$ & $\begin{array}{l}115 \\
105\end{array}$ & $\begin{array}{l}11 \\
6\end{array}$ & $\begin{array}{l}74 \\
68\end{array}$ & $\begin{array}{l}71 \\
67\end{array}$ & $\begin{array}{l}3 \\
1\end{array}$ \\
\hline $\begin{array}{l}\text { Schneid } \\
\text { er et al }\end{array}$ & $\begin{array}{l}\text { Stress } \\
\text { reduction on } \\
\text { mortality. } \\
\text { Pooled data- } \\
\text { TM, MM, } \\
\text { mental } \\
\text { relaxation }\end{array}$ & $\begin{array}{l}>18 \text { year } \\
\text { s for } \\
\text { white } \\
\text { and } 9 \\
\text { years } \\
\text { for } \\
\text { black }\end{array}$ & $\begin{array}{l}202 \\
\text { Whit } \\
\text { e } 77 \\
\text { Blac } \\
\text { k } \\
125\end{array}$ & $\begin{array}{l}\text { TM 21, } \\
\text { MM23 } \\
\text { Ment R } \\
\text { 22, C } 11 \\
\text { TM 40, } \\
\text { PMR } 42 \\
\text { Health } \\
\text { edu. } 43\end{array}$ & $\begin{array}{l}\text { M-14 } \\
\text { F-63 } \\
\text { M-54 } \\
\text { F-71 }\end{array}$ & $\begin{array}{l}\text { White } \\
>85 \\
\text { years } \\
\text { Blacks } \\
>66 \\
\text { years }\end{array}$ & $\begin{array}{l}\geq 140 / 90 \\
\text { To } 160 / 100\end{array}$ & & & $\begin{array}{l}\text { Redu } \\
\text { ction } \\
\text { in } \\
\text { SBP/ } \\
\text { DBP }\end{array}$ & & & \\
\hline
\end{tabular}

On analysis of these studies we observe in this table reduction in systolic blood pressure ranging from 10-26mmHg in meditators compared to $2-6 \mathrm{mmHg}$ in control groups and reduction in diastolic blood pressure ranging from 4-13 mm $\mathrm{Hg}$ meditators compared to 1$4 \mathrm{mmHg}$ in non-meditators.

Table 3: Standardized mean differences in systolic and diastolic blood pressure changes after meditation compared to control.

\begin{tabular}{|c|c|c|c|c|c|c|c|c|c|c|}
\hline $\begin{array}{l}\text { Author } \\
\text { Name }\end{array}$ & Title & $\begin{array}{l}\text { Sample } \\
\text { size }\end{array}$ & $\begin{array}{l}\text { Study } \\
\text { group } \\
\text { /control }\end{array}$ & $\begin{array}{l}\text { Cut off } \\
\text { Point } \\
\text { mmHg }\end{array}$ & SBP (B) & $\operatorname{SBP}(\mathbf{A})$ & $\begin{array}{l}\text { SBP } \\
(B-A)\end{array}$ & $\begin{array}{l}\text { DBP } \\
\text { (B) }\end{array}$ & DBP (A) & $\operatorname{DBP}(\mathbf{B}-\mathbf{A})$ \\
\hline $\begin{array}{l}\text { Schneider } \\
\text { RH et al }\end{array}$ & $\mathrm{TM}$ in $\mathrm{HTN}$ & 74 & $\begin{array}{l}36 \\
38 \\
\end{array}$ & $\begin{array}{l}>140 / 90 \\
\text { to } 180 / 110\end{array}$ & $\begin{array}{l}145.4 \underline{+} 12.3 \\
150.4 \underline{+} 14.3\end{array}$ & $\begin{array}{l}135 \pm 1.6 \\
149.9^{ \pm} 0.6\end{array}$ & $\begin{array}{l}10.4 \underline{+1.6} \\
1.57 \pm 2.7\end{array}$ & $\begin{array}{l}93.7+9.3 \\
91.7+9.2\end{array}$ & $\begin{array}{l}88 \pm 1.2 \\
90.9+1.6\end{array}$ & $\begin{array}{l}10.4+2.1 \\
0.8 \pm 1.6\end{array}$ \\
\hline $\begin{array}{l}\text { Schneider } \\
\text { RH et al }\end{array}$ & TM in HTN & 106 & $\begin{array}{l}54 \\
52 \\
\end{array}$ & $>140 / 95$ & $\begin{array}{l}142.1 \pm 13.5 \\
144.4 \pm 17.2\end{array}$ & $\begin{array}{l}139 \pm 1.52 \\
143.5 \pm 1.71\end{array}$ & $\begin{array}{l}3 \pm 0.4 \\
0.9 \pm 0.12\end{array}$ & $\begin{array}{l}95.1 \pm 4 \\
95.7 \pm 3.6\end{array}$ & $\begin{array}{l}89 \pm 0.89 \\
95 \pm 1\end{array}$ & $\begin{array}{l}6 \pm 0.86 \\
0.7 \pm 0.097\end{array}$ \\
\hline $\begin{array}{l}\text { Peter seer } \\
\text { et al }\end{array}$ & TM in HTN & 41 & & & & & & & & $\begin{array}{l}\text { Reduction } \\
\text { in DBP }\end{array}$ \\
\hline \multirow[t]{2}{*}{$\begin{array}{l}\text { Priya } \\
\text { Palta et al }\end{array}$} & $\begin{array}{l}\text { MM in afro - } \\
\text { Am low income }\end{array}$ & 20 & 12 & & $148.5+17.5$ & $137.5 \pm 16.1$ & $11 \pm 3.17$ & $78 \pm 9.5$ & $74.5 \pm 11.2$ & $4 \pm 1.54$ \\
\hline & $\begin{array}{l}\text { Gp. Include pt } \\
\text { on medicine }\end{array}$ & & 8 & & $144.5 \pm 27.7$ & $140.3 \pm 15.8$ & $4 \pm 1.41$ & $67.3 \pm 13.4$ & $70.3+9.1$ & $+3 \pm 1.06$ \\
\hline \multirow{2}{*}{$\begin{array}{l}\text { JP } \\
\text { Manikond } \\
\text { a et al }\end{array}$} & $\begin{array}{l}\text { Contemplative } \\
\text { meditation and }\end{array}$ & 49 & 26 & $\begin{array}{l}>140 / 85 \text { to } \\
180 / 110\end{array}$ & 168 & 153 & $15 \pm 2.94$ & 108 & 97 & $13 \pm 1.80$ \\
\hline & $\begin{array}{l}\text { Breath } \\
\text { Technique in } \\
\text { HTN }\end{array}$ & & 23 & & 167 & 164 & $3 \pm 0.62$ & 107 & 103 & $4 \pm 0.78$ \\
\hline \multirow[t]{2}{*}{$\begin{array}{l}\text { Kimberly } \\
\text { et al }\end{array}$} & MM in HTN & 101 & 51 & $\begin{array}{l}\text { Amb.BP } \\
135 / 85\end{array}$ & 140 & 139.6 & $0.4+6.7$ & 87 & 86.6 & $0.0 \pm 4.9$ \\
\hline & & & 50 & $\begin{array}{l}\text { 24hours } \\
>130 / 80\end{array}$ & -- & & $0.4 \pm 7.8$ & -- & -- & $0.0 \pm 4.6$ \\
\hline $\begin{array}{l}\text { Marcia de } \\
\text { Fatima } \\
\text { Rosas } \\
\text { Marchiori } \\
\text { et al }\end{array}$ & $\begin{array}{l}\text { Zen Meditation } \\
\text { in HTN. } \\
\text { Patients } \\
\text { stabilized on } \\
\text { drugs included }\end{array}$ & 59 & $\begin{array}{l}28 \\
31\end{array}$ & $\begin{array}{l}130 / 85 \text { to } \\
160 / 100\end{array}$ & & & & & $\begin{array}{l}\text { Reduction } \\
\text { in DBP }\end{array}$ & $\begin{array}{l}\text { Reduction } \\
\text { in } \\
\text { diastolic } \\
\text { blood } \\
\text { pressure }\end{array}$ \\
\hline $\begin{array}{l}\text { Mahdy } \\
\text { Hasanzad } \\
\text { ah }\end{array}$ & $\begin{array}{l}\text { MM in CVD } \\
\text { and depression } \\
\text { (card rehab. } \\
\text { progm) }\end{array}$ & 45 & $\begin{array}{l}\text { MM 15 } \\
\text { PMR } 15 \\
\text { CTR } 15\end{array}$ & & $\begin{array}{l}134.4^{ \pm 17.8} \\
126.4 \pm 17 \\
111.4 \pm 14.9\end{array}$ & $\begin{array}{l}107.9 \pm 10.1 \\
115.1 \pm 11.8 \\
105.1 \pm 13.2\end{array}$ & $\begin{array}{l}26 \pm 6.71 \\
11 \\
6 \pm 1.54\end{array}$ & $\begin{array}{l}71.7 \pm 15.5 \\
74.9 \pm 6.9 \\
68 \pm 8\end{array}$ & $\begin{array}{l}66.7 \pm 7.6 \\
71.9 \pm 6.9 \\
66.7 \pm 7.6\end{array}$ & $\begin{array}{l}5 \pm 1.7673 \\
1.3 \pm 0.35\end{array}$ \\
\hline
\end{tabular}

Table 4: Standardized mean difference (SMD) of individual studies (systolic blood pressure).

\begin{tabular}{|c|c|c|c|c|}
\hline Author & Study & SMD & $95 \% \mathrm{CI}$ & Variance (v) \\
\hline Schneider RH et al & TM in hypertension & -3.95 & -3.17 to -4.7 & -0.16 \\
\hline Schneider RH et al & TM in Hypertension & -7.05 & -6.03 to -8.07 & -0.27 \\
\hline Priya Palta et al & MM in Afro-Am low income Gp. Include pt on medicine & -2.66 & -1.44 to -3.87 & -0.38 \\
\hline JP Manikonda et al & Contemplative meditation \& breath technique in hypertension & -5.49 & -4.26 to -6.71 & -0.38 \\
\hline Kimberly et al & Mindfulness meditation in Hypertension & 0 & -0.39 to -0.38 & -0.38 \\
\hline Mahdy Hasanzadah & MM in CVD \& depression (card rehab. programme) & -4.10 & -2.84 to -5.36 & -0.44 \\
\hline
\end{tabular}

\section{The variance weighted mean difference (Meta-analysis) (Table 4 and 5)}

- $\quad$ Standardized mean difference (d) for systolic blood pressure (SBP) six of 8 studies: $d=0.8548 ; 95 \% \mathrm{CI}$, 0.6446 to $-1.0649 ; \mathrm{v}=0.0115$.
- $\quad$ Standardized mean SBP difference for 5 of 8 studies (excludes Kinberley et al study): $d=1.40 ; 95 \%$ CI, 1.1396 to- $1.6638 ; \mathrm{v}=0.0179$.

- Standardized mean difference for diastolic blood pressure (DBP) for 6 of 8 studies: $d=1.9685$; $95 \%$ $\mathrm{CI}=-2.2852$ to $-1.6518 ; \mathrm{v}=0.261$. 
- $\quad$ Standardized mean difference for DBP for 5 of 8 studies (excludes Kimberley et al): d=1.9685; $95 \%$ CI, -2.2852 to -1.6518 ; $v=0.0218$.

Table 5: Standardized mean difference (SMD) of individual studies (diastolic blood pressure).

\begin{tabular}{|c|c|c|c|c|}
\hline Author & Study & SMD & $\begin{array}{l}95 \% \\
\text { CI }\end{array}$ & $\begin{array}{l}\text { Variance } \\
\text { (v) }\end{array}$ \\
\hline $\begin{array}{l}\text { Schneider } \\
\text { RH et al }\end{array}$ & TM in hypertension & -3.73 & $\begin{array}{l}-2.98 \\
\text { to } 4.49\end{array}$ & -0.16 \\
\hline $\begin{array}{l}\text { Schneider } \\
\text { RH et al }\end{array}$ & TM in Hypertension & -8.46 & $\begin{array}{l}-7.26 \\
\text { to } \\
-9.66\end{array}$ & -0.37 \\
\hline $\begin{array}{l}\text { Priya Palta } \\
\text { et al }\end{array}$ & $\begin{array}{l}\text { MM in Afro-Am low } \\
\text { income Gp. Include } \\
\text { pt on medicine }\end{array}$ & +0.89 & $\begin{array}{l}-0.04 \\
\text { to } \\
-1.83\end{array}$ & -0.22 \\
\hline $\begin{array}{l}\text { JP } \\
\text { Manikonda } \\
\text { et al }\end{array}$ & $\begin{array}{l}\text { Contemplative } \\
\text { meditation \& breath } \\
\text { technique in } \\
\text { hypertension }\end{array}$ & -9.12 & $\begin{array}{l}-7.2 \text { to } \\
-11\end{array}$ & 0.93 \\
\hline $\begin{array}{l}\text { Kimberly } \\
\text { et al }\end{array}$ & $\begin{array}{l}\text { Mindfulness } \\
\text { meditation in } \\
\text { Hypertension }\end{array}$ & 0 & $\begin{array}{l}-0.39 \\
\text { to } \\
+0.39\end{array}$ & -0.39 \\
\hline $\begin{array}{l}\text { Mahdy } \\
\text { Hasanzadah }\end{array}$ & $\begin{array}{l}\text { MM in CVD \& } \\
\text { depression(card } \\
\text { rehab. programme) }\end{array}$ & -2.37 & $\begin{array}{l}-1.44 \\
\text { to } \\
-3.31 \\
\end{array}$ & 0.22 \\
\hline
\end{tabular}

\section{Analysis}

After going through abstract of more than 55 studies we took out full articles of 10 studies which included study on effect of meditation on cardio-vascular diseases especially Hypertension or meditation is one of the components included as influencing factors in Hypertension. Eight studies were randomised control trial (3 on TM in hypertension with follow up period of 3 months, 1 on TM in mortality reduction in hypertension with follow-up period of $>9$ years; 3 on Mindfulness with follow up period of 2 months; 1 on contemplative meditation with follow up period of 2 months and 1 on Zen having follow up period of 3 months).

Two studies were experimental and 2 on normal adult populations involving selective sampling (DhammakayaThailand \& Islamic Meditation-Malaysia).

Islamic meditation and Dhammakaya meditation does not meet our inclusion criteria because the duration of follow up was short and study group included normal individuals, However it must be noted that Islamic meditation found reduction in systolic blood pressure of $3 \mathrm{~mm}$ of $\mathrm{Hg}$ and diastolic blood pressure of $2 \mathrm{mmHg}$ after Salat; also found reduction in heart rate; and Dhammakaya meditation found reduction in blood pressure and serum cortisol level. ${ }^{33,36}$

All studies show reduction in systolic blood pressure after meditation ranging from $10-26 \mathrm{mmHg}$ systolic compared to 2-6 $\mathrm{mmHg}$ in control group and $3-13 \mathrm{mmHg}$ diastolic blood pressure compared to $1-4 \mathrm{mmHg}$ in control group (Table 2). The variance-weighted mean difference (MetaAnalysis) in systolic blood pressure in 6 studies was -0.85
(95\% CI, -0.64 to -1.06$)$ and in five studies was $1.4(95 \%$ $\mathrm{CI},-1.1396$ to -1.66$)$; whereas standardized mean difference for diastolic blood pressure for six studies was $-2.49(95 \% \mathrm{CI},-2.1798$ to -2.8$)$ and for 5 studies -1.78 (95\% CI, -1.5 to -2.06 ) (Table 3 ).

In all the meditation technique it was observed that meditation practitioners had reduced anxiety and psychological well-being. ${ }^{20,21}$ Studies also show reduction cardiovascular and all-cause mortality. .Another relevant study is by Robert H Schneider et al published in 2012, randomized controlled trial on 201 black men $(n=117)$ and women $(n=84)$, mean age 59 years with CAD in which effect of transcendental meditation was compared with health education. Patients were followed in two phases 1998-2003 and 2004-2007, with average follow up period of 5.4 years. This study found $48 \%$ risk reduction of all-cause mortality, non-fatal MI and stroke in TM patients compared with health education.

\section{DISCUSSION}

In meditation, the mind calms down, body goes into state of hypo-metabolism, muscles relax, and physiological functions normalize. Studies on CVS found decreased peripheral vascular resistance, increased elasticity or normalization of elasticity of arterial muscle fibres and improved blood supply to various organs / tissues. Strong motivation and determination brings about positive change in attitude and behaviour. ${ }^{3,23}$ During meditation, the mind goes into a state similar to that of deep sleep, but the meditator remains fully conscious, awake, alert and responsive. $^{17}$

At mental level there is increased clarity of thoughts and perception and person is emotionally stable. At hormonal level production of cortisol and catecholamine is reduced. ${ }^{16,20,33}$ With regular practice of meditation autonomic nervous system is well regulated. This is because of control over parasympathetic activity by anterior cingulate cortex. ${ }^{18,25}$ Thus meditation brings about physiological changes, neuro-hormonal changes and behavioural changes. Under these conditions, a meditating person is fully awakened, is at peace and in happy state of mind.

\section{Beneficial effects of meditation}

Cardiovascular effects seen in meditation are reduction in pulse rate, heart rate, stroke volume, vascular resistance. The behaviour of heart rate changes from high complex to low-dimensional chaotic motions. ${ }^{26,27}$ At cellular level oxygen $\left(\mathrm{O}_{2}\right)$ consumption and carbon dioxide $\left(\mathrm{CO}_{2}\right)$ elimination is reduced. Respiratory system shows fall in respiratory rate and minute ventilation volume with no change in respiratory quotient and in CNS blood supply and perfusion of cerebral cortex increases. ${ }^{23,24}$ The meditator feels cheerful and happy, adapts better with situation and able to deal with problems courageously. ${ }^{5}$ 


\section{How the intervention can help in preventing hypertension?}

Studies have shown that acute and chronic psychological stress, anxiety, depression, hostility and anger contributes to hypertension, whereas optimal level of melatonin causes good sleep and reduces stress level and blood pressure is normalized or reduced. ${ }^{28}$ Meditation prevents excessive release of hormones like Cortisol, Epinephrine and increases production of DHEA, Melatonin, Serotonin, TSH, Human growth hormone and Endorphins. ${ }^{14,28-30}$

Meditation and discourses which follows after meditation help understand and removal of psychological barriers and negativities; inculcate positive thoughts and emotions thereby lead to physiologically quietened state, better tolerance and adaptability in different situations. ${ }^{31,32}$ By reducing sympathetic tone and developing parasympathetic dominance; reduction of stress hormones; meditation reduces blood pressure, improves cardiac perfusion, reduce future angina, arrhythmias and speed up time to return home and on job. ${ }^{33-35}$

When psycho-social interventions were added to standard medical treatment, significant improvement was noticed in term of survival benefits and aversion of cardiac events. ${ }^{36}$ In view of these beneficial effects, meditation can be included as primary intervention measure for prevention of hypertension and also integrated into cardiac rehabilitation programme.

\section{Adverse effects}

There were no known adverse effects of meditation.

\section{Limitations}

Most of studies are on ambulatory basis and duration of therapy varies. In some studies, it is 20 minutes morningevening for 3 months and others for 30-45 minutes twice daily for 2 months. Moreover sample sizes are also small. These factors may affect meditation outcomes in hypertension. We feel more studies need to be done on larger sample under controlled environment.

\section{CONCLUSION}

Persistence stress has been found to be associated with rise in blood pressure because of increased release of catecholamine and corticoids. Meditation is a mind control technique which help individual to reduce stress. When mind is at peace sympathetic output is low and there is parasympathetic dominance. This helps reduce blood pressure, prevent hypertension and complications associated with hypertension; reduced cardiovascular morbidity and mortality. Also person feels relieved of anxiety and depression and generally there is a sense of inner peace.
Funding: No funding sources

Conflict of interest: None declared

Ethical approval: Not required

\section{REFERENCES}

1. Rampal S, Azhar MZ, Rahman AR. Prevalence, awareness, treatment and control of Hypertension in Malaysia: A national study of 16,440 subjects. Public Health. 2008;122(10):11-8.

2. Shukla AN, Madan T, Thakkar BM, Parmar MM, Shah KH. Prevalence and Predictors of Undiagnosed Hypertension in an Apparently Healthy Western Indian Population; Advances in Epidemiology. 2015;(2015), Article ID 649184, 5 pages.

3. Cathleen D. Gillespie, MS1 Kimberly A. Hurvitz, MHS2. Prevalence of Hypertension and Controlled Hypertension - United States, 2007-2010; Centre for Disease Control and Prevention; Morbidity and Mortality Weekly Report; Supplements. 2013;62(03);144-8.

4. The buddhist centre: buddhism for today. https://thebuddhistcentre.com/text/what-meditation

5. Manar M, Nabolsi RN, Alexander M, Carson RN. Spirituality, illness and personal responsibility: the experience of Jordanian Muslim men with coronary artery disease; Scandinavian Journal of Caring Sciences. 2011;25(4):716-24.

6. Ziegelstein RC. Acute Emotional Stress \& Cardiac Arrhythmia. JAMA. 2007;298(3):324-9.

7. Schwartz BG, Mayeda GS, Burstein S, Economides $\mathrm{C}$, Kloner RA. When and why do heart attacks occur? Cardiovascular Triggers and Their Potential Role. Hosp Pract Minneap. 2010;38(5):144-52.

8. Ornish D, Scherwitz LW, Billings JH, Brown SE, Gould KL, Merritt TA, et.al. Intensive lifestyle changes for reversal of coronary heart disease. JAMA. 1998; 280(23):2001-7.

9. Simula S, Vanninen E, Viitanen L, Kareinen A, Lehto S, Pajunen P, et. al. Cardiac Adrenergic Innervation is affected in Asymptomatic Subjects with Very Early Stage of Coronary Artery Disease. Journal of Nuclear Medicine. 2002;43(1):1-7.

10. Esler M. The autonomic Nervous System \& Cardiac Arrhythmias. Clinical Autonomic Research. 1992;2(2):133-5.

11. Gaziano TA, Reddy KS, Paccaud F, Horton S, Chaturvedi V. Cardiovascular Disease. Geneva: The World Health Organization. 2002.

12. Deedwania PC, Carbajal EV. Silent ischemia during daily life is an independent predictor of mortality in Stable Angina. Circulation. 1990;81(3):748-56.

13. Paul-Labrador M, Polk D, Dwyer JH, Velasquez I, Nidich S, Rainforth M, et al. Effects of a Randomized Controlled Trial of Transcendental Meditation on Components of the Metabolic Syndrome in Subjects With Coronary Heart Disease Arch Intern Med. 2006;166(11):1218-24. 
14. Curiati JA, Bocchi E, Freire JO, Arantes AC, Braga M, Garcia Y, et al. Meditation reduces sympathetic activation and improves quality of life in elderly patients with optimally treated heart failure: A prospective randomised study; J. Alt. and Compl. Med. 2005;11(3);465-72.

15. William H. Art of living: Vipassana Meditation: As taught by S.N. Goenka. New York: Harper Collins Publishers. 1987.

16. Ahsan A, Khan M, Siddhique R. The Healing Power of prayer in Islam; Indian J. Positive Psychology. 2012;3(2):168-72.

17. Wallace RK, Benson H. A wakeful hypometabolic physiologic state. American Journal of Physiology. 1971; 221: 795-9.

18. Tang Y, Yinghua M, Fan Y, Feng H, Wang J, Ye Zhang $\mathrm{S}$, et al. Central and autonomic nervous system interaction is altered by short-term meditation; Proc Natl Acad Sci USA. 2009;106(22):8865-70.

19. Doufesh H, Ibrahim F, Ismail NA. Assessment of heart rate and blood pressure in different Salat Positions; J. Phys. The.Sci. 2013;25:211-4.

20. Marchiori MFR, Kozasa EH, Miranda RD, Andrade ALM, Perrotti TC, Leite JR. Decrease in blood pressure and improved psychological aspects through meditation training in hypertensive older adults: A randomized control study; Geriatrics \& Gerontology International. 2014;15(10):1158-64.

21. Delui MH, Yari M, khouyinezhad G, Amini M, Bayazi MH. Comparison of Cardiac Rehabilitation Programs Combined with Relaxation and Meditation Techniques on Reduction of Depression and Anxiety of Cardiovascular Patients. The Open Cardiovascular Medicine Journal. 2016(10).

22. Schneider RH, Grim CE, Rainforth MV, Kotchen T, Nidich SI, Gaylord-King C, et al. Stress Reduction in the Secondary Prevention of Cardiovascular Disease; Circulation: Cardiovascular Quality and Outcomes. 2012;5:750-8.

23. Jevning R, Wallace RK, Beidebach $M$. The physology of meditation: A review. A wakeful hypometabolic integrated response. Neurosci. Biobehav. Rev. 1992;16:415-24.

24. Sudsuang R. Effect of buddhist meditation on serum cortisol and total protein levels, blood pressure, pulse rate, lung volume and reaction time; Physiology \& Behavior. 1991;50(3):543-8.

25. Newton $A B$, Iversen $J$. The neural basis of the complex mental task of meditation. Neurotransmitter and Neurochemical considerations. 2003;61(2):283-91.

26. Herrmann JM. Essential hypertension and stress: When do yoga, psychotherapy and autogenic training help? MMW Fortschr Med. 2002;144(19):38-41.

27. Ateke Goshvarpour IJ. Image, Graphics and Signal Processing. MECS. 2012;2:44-50. (http://www.mecs-press.org/).
28. Brzezinski A. Mechanisms of disease: melatonin in humans. New England Journal of Medicine. 1997;336:186-95.

29. MacLean CRK, Walton KG, Wenneberg SR, Levitsky DK, Mandarino JP, Waziri R, et al. Schneider. Effects of the transcendental meditation program on adaptive mechanisms: Changes in hormone levels and responses to stress after 4 months of practice, Psycho-neuro-endocrinology. 1997;22(4):277-95.

30. MacLean CR, Walton KG, Wenneberg SR, Levitsky DK, Mandarino JP, Waziri R, et. al. Effect of TM programme on adaptive mechanisms: Changes in hormonal levels and responses to stress after four months practice. Psycho-neuroendocrinology. 1997;22:277-95.

31. Porges SW. Cardiac vagus tone: a physiological index of stress. Neurosciences and Behavioural Review. 1995;19(2):225-3.

32. David S, Ludwig JKZ. Clinical mindfulness and Patient safety. JAMA. 2010;304(22);2532-33.

33. White VD. Relaxation Therapy for rehabilitation and prevention in Ischemic heart disease: a systematic review and meta-analysis. Euro $\mathbf{J}$ cardiovasc prev Rehabili. 2005;12(3):193-202.

34. Zamarra JW, Schneider RH, Besseghini I, Robinson DK, Salerno JW. Usefulness of Transcendental Meditation Programme in the treatment of coronary artery disease. Am J Cardiol. 1996:15;77(10):86770.

35. Gupta SK, Sawhney RC, Rai L, Chavan VD, et al. Regression of coronary atherosclerosis through healthy lifestyle in coronary artery disease patientsMount Abu Open heart Trial; Indian Heart J. 2011;63:461-69.

36. Linden W, Stossel C, Maurice J. Psychosocial Interventions for patients with coronary artery disease: A meta-analysis. Arch Intern Med. 1996;156(7):745-52.

37. Schneider RH, Staggers F, Alxander CN, Sheppard W, Rainforth M, Kondwani K, et al. A randomised controlled trial of stress reduction for hypertension in older African Americans. Hypertension. 1995;26(5):820-7.

38. Schneidera RH, Alexandera $\mathrm{CN}$, Staggersb F, Orme-Johnsona DW, Rainfortha M, Salernoa JW, et al. A randomized controlled trial of stress reduction in African Americans treated for hypertension for over one year Portions of these results were presented at the American Heart Association Scientific Sessions. American Journal of Hypertension. 2005;18(1):88-98.

39. Schneider RH, Alexander CN, Staggers F, Rainforth M, Salerno JW, Hartz A, et al. Long-Term Effects of Stress Reduction on Mortality in Persons $\geq 55$ Years of Age with Systemic Hypertension; American Journal of Cardiology. 2005;1;95(9):1060-4. 
40. Seer P, Raeburn JM. Meditation Training and essential hypertension: A methodological study. Journal of behavioural Medicine. 1980;3:59-71.

41. Palta P, Piferi RL, Gill JM, Hayat MJ, Connolly AB, Szanton SL. Evaluation of a MindfulnessBased Intervention Program to Decrease Blood Pressure in Low-Income African-American Older Adults; J Urban Health. 2012;89(2):308-16.

42. Manikonda JP, Störk S, Tögel S, Lobmüller A, Grünberg I, Bedel $S$, et al. Contemplative meditation reduces ambulatory blood pressure and stress-induced hypertension: a randomized pilot trial. J Hum Hypertens. 2008;22(2):138-40.

43. Blom K, Baker B, How M, Dai M, Irvine J, Abbey $\mathrm{S}$, et al. Hypertension Analysis of Stress Reduction Using Mindfulness Meditation and Yoga: Results From the Harmony Randomized Controlled Trial; Am J Hypertens. 2014;27(1):122-9.

Cite this article as: Khobragade $\mathrm{Y}$, Khobragade $\mathrm{S}$, Abbas AL. Hypertension and meditation: can meditation be useful in preventing hypertension? Int $\mathbf{J}$ Community Med Public Health 2016;3:1685-94. 